\title{
Collatz cycles with few descents
}

\author{
by
}

\section{T. Brox (Stuttgart)}

1. Introduction. Let $T: \mathbb{Z} \rightarrow \mathbb{Z}$ be the function defined by $T(x)=x / 2$ if $x$ is even, $T(x)=(3 x+1) / 2$ if $x$ is odd. $T$ is known as the $3 x+1$ function (see [4], [7]). We are interested in the cycles of $T$, referred to as $3 x+1$ cycles (see [3]-[5]). The well known Finite Cycle Conjecture asserts that $T$ has only finitely many cycles. In this note we generalize a theorem due to R. Steiner (see [4], [6]).

In order to state the result in a concise form, we consider instead of $T$ a slightly different function $T_{1}$ defined by the unique decomposition

$$
2^{k(x)} T_{1}(x)=3 x+1 \quad(x \text { odd }),
$$

where $k(x) \geq 1$ is the multiplicity of the prime factor 2 in the number $3 x+1$. Note that $T_{1}$ is only defined for odd integers. For each odd argument the iteration $T^{k(x)}(x)$ is equal to $T_{1}(x)$. An odd integer $x$ is called descending if $k(x) \geq 2$.

In what follows, a cycle of $T_{1}$ is called a Collatz cycle. By definition, we can represent a Collatz cycle by a tuple $\Gamma=\left(x_{1}, \ldots, x_{n}\right)$ consisting of distinct odd integers $(n \geq 1)$. By $|\Gamma|$ we denote the number of elements in $\Gamma$, i.e. the period of the Collatz cycle. Each Collatz cycle consists of the odd elements in a $3 x+1$ cycle, and conversely. If $k$ denotes the sum of $k(x)$ over all elements of a Collatz cycle, then $k$ is the period of the corresponding $3 x+1$ cycle. Let $\delta(\Gamma)$ be the number of descending elements in a Collatz cycle $\Gamma$.

TheOREM 1.1. The number of Collatz cycles satisfying $\delta(\Gamma)<2 \log |\Gamma|$ is finite.

We will prove Theorem 1.1 in the following sections. In Section 4 we briefly discuss the extension of Theorem 1.1 to $3 x+d$ mapping (see [2]). The theorem of R. Steiner states that the fixed point 1 is the only positive

2000 Mathematics Subject Classification: 11D61, $11 \mathrm{Z} 05$. 
Collatz cycle with $\delta(\Gamma)=1$. It is easy to deduce the following corollary of Theorem 1.1, using Lemma 2.2 in Section 2.

COROLlary 1.2. For each fixed $\nu \geq 1$ the number of Collatz cycles with $\delta(\Gamma) \leq \nu$ is finite.

2. A divisibility problem. In this section we express the existence problem of a Collatz cycle by an appropriate periodic sequence (see Sections 2,5 of [3]). Given an odd integer $x_{0}$, consider the iteration $x_{m}=T_{1}\left(x_{m-1}\right)$. Let $k_{m}=k\left(x_{m-1}\right)$. Because of (1.1), we get

$$
2^{k_{m}} x_{m}=3 x_{m-1}+1 \quad(m \geq 1),
$$

where it is required that $x_{1}, x_{2}, \ldots$ are odd. Proceeding iteratively, we immediately obtain

$$
2^{k_{1}+\ldots+k_{m}} x_{m}=3^{m} x_{0}+\varphi_{m-1}\left(k_{1}, \ldots, k_{m-1}\right) .
$$

Here $\varphi_{0}=1$ and

$$
\varphi_{m}\left(u_{1}, \ldots, u_{m}\right)=\sum_{l=0}^{m} 3^{m-l} 2^{u_{1}+\ldots+u_{l}} \quad\left(\left(u_{1}, \ldots, u_{m}\right) \in \mathbb{R}^{m}\right),
$$

where $u_{1}+\ldots+u_{l}$ is zero if $l=0$. For further references see Section 2 of [3].

Assume now $\left(x_{0}, \ldots, x_{n-1}\right)$ is a Collatz cycle. By construction, $k_{i} \geq 1$ for $1 \leq i \leq n$. Since $x_{n}=x_{0},(2.2)$ implies

$$
M x_{0}=\varphi_{n-1}\left(k_{1}, \ldots, k_{n-1}\right),
$$

where

$$
M=2^{k}-3^{n}, \quad k=k_{1}+\ldots+k_{n} .
$$

Consider $\left(x_{0}, \ldots, x_{n-1}\right)$ and $\left(k_{1}, \ldots, k_{n}\right)$ as $n$-periodic sequences $\left\{x_{i}\right\}_{i \in \mathbb{Z}}$, $\left\{k_{i}\right\}_{i \in \mathbb{Z}}$. Since we can take each $x_{i}$ as start value, we obtain

$$
M x_{i}=\varphi_{n-1}\left(k_{i+1}, \ldots, k_{i+n-1}\right) \quad(i \in \mathbb{Z}) .
$$

Hence $M$ divides the right hand side of (2.5) for each $i$. Since the Collatz cycle consists of distinct elements, $n$ is the smallest period of $\left\{k_{i}\right\}$, by (2.5).

Conversely, let $\left\{k_{i}\right\}_{i \in \mathbb{Z}}$ be any periodic sequence of positive integers. Let $n$ be the smallest period of $\left\{k_{i}\right\}$. Define $M$ and $k$ by (2.4). Let

$$
F_{i}=\varphi_{n-1}\left(k_{i+1}, \ldots, k_{i+n-1}\right) \quad(i \in \mathbb{Z}) .
$$

Note that $F_{i}$ does not depend on $k_{i}$. Plainly $\left\{F_{i}\right\}$ is $n$-periodic. Because of (2.3) and periodicity, we get by a simple computation

$$
2^{k_{i}} F_{i}=3 F_{i-1}+M \quad(i \in \mathbb{Z}) .
$$


Since each $k_{i}$ is positive, we conclude from (2.3) and (2.4) that

$$
\begin{gathered}
\operatorname{gcd}\left(2 \cdot 3, F_{i}\right)=1 \quad(i \in \mathbb{Z}), \\
\operatorname{gcd}(2 \cdot 3, M)=1 .
\end{gathered}
$$

Because of (2.6) and (2.7), $k_{i}$ and $F_{i}$ are uniquely determined by $F_{i-1}$. If $F_{l}=F_{m}$, then $|m-l|$ is a period of $\left\{k_{i}\right\}$. Hence $F_{1}, \ldots, F_{n}$ are distinct. We are now ready to prove the following lemmas (see also Section 5 of [3]).

Lemma 2.1. Let $M=2^{k}-3^{n}$. Then either $M \nmid F_{i}(1 \leq i \leq n)$ or $M \mid F_{i}(1 \leq i \leq n)$. The latter case corresponds to a Collatz cycle $\left(x_{1}, \ldots, x_{n}\right)$, where $x_{i}=F_{i} / M$.

Proof. Assume that $M \mid F_{i}$ for one $i$. Then (2.6)-(2.8) imply that for all $i, M \mid F_{i}$ and $x_{i}=F_{i} / M$ is odd. Dividing (2.6) by $M$, we arrive at $x_{i}=T_{1}\left(x_{i-1}\right)$, since each $x_{i}$ is odd. Plainly $x_{1}, \ldots, x_{n}$ are distinct.

Lemma 2.2. If $\left\{k_{i}\right\}$ generates a Collatz cycle, then $k=k_{1}+\ldots+k_{n} \leq 2 n$. In particular, for each $n \geq 1$, the number of Collatz cycles with $|\Gamma|=n$ is finite.

Proof. If $\left\{k_{i}\right\}$ generates a Collatz cycle, then $x_{i}=F_{i} / M$, by Lemma 2.1. If we multiply (2.6) from $i=1$ to $i=n$, a simple reduction yields

$$
2^{k}=\prod_{i=1}^{n}\left(3+1 / x_{i-1}\right) \leq 4^{n},
$$

since $\left|1 / x_{i}\right| \leq 1$. Hence $k \leq 2 n$.

Remark 2.3. By Lemma 2.1, since $k_{i}=1(i \in \mathbb{Z})$ has period 1 , there exists at most one Collatz cycle with $\delta(\Gamma)=0$, and this Collatz cycle has to be a fixed point. In fact, $-1=1 /(2-3)$ is a non-descending fixed point.

3. Algebraic reformulation. In order to prove Theorem 1.1, we reformulate the divisibility problem in a more convenient form. As before let $\left\{k_{i}\right\}_{i \in \mathbb{Z}}$ be a periodic sequence of positive integers. Let $n$ be the smallest period of $\left\{k_{i}\right\}$. Define

$$
\begin{array}{r}
\widetilde{\varphi}_{n}\left(u_{1}, \ldots, u_{n}\right)=2^{u_{1}} \varphi_{n-1}\left(u_{2}, \ldots, u_{n}\right)-2 \varphi_{n-1}\left(u_{1}, \ldots, u_{n-1}\right) \\
\left(\left(u_{1}, \ldots, u_{n}\right) \in \mathbb{R}^{n}\right) .
\end{array}
$$

Because of (2.3), we obtain

$$
\widetilde{\varphi}_{n}\left(u_{1}, \ldots, u_{n}\right)=2 \sum_{l=0}^{n-1} 3^{n-(l+1)} 2^{u_{1}+\ldots+u_{l}}\left\{2^{u_{l+1}-1}-1\right\} .
$$

For each $i \in \mathbb{Z}$ define

$$
\widetilde{F}_{i}=\widetilde{\varphi}_{n}\left(k_{i+1}, \ldots, k_{i+n}\right) .
$$


Then (2.6) implies

$$
\widetilde{F}_{i}=2^{k_{i+1}} F_{i+1}-2 F_{i}=F_{i}+M \quad(i \in \mathbb{Z}) .
$$

Let $\nu$ be the number of indices $i$ such that $1 \leq i \leq n$ and $k_{i} \geq 2$. We assume $\nu \geq 1$. Define strictly ascending numbers $\tau(1), \ldots, \tau(\nu)$ by $1 \leq$ $\tau(j) \leq n$ and $k_{\tau(j)} \geq 2$. Put $\tau(0)=\tau(\nu)-n$. For $1 \leq j \leq \nu$ let

$$
h_{j}=k_{\tau(j)}-1, \quad n_{j}=\tau(j)-\tau(j-1) .
$$

Consider $\left(h_{1}, \ldots, h_{\nu}\right),\left(n_{1}, \ldots, n_{\nu}\right)$ as $\nu$-periodic sequences $\left\{h_{i}\right\}_{i \in \mathbb{Z}},\left\{n_{i}\right\}_{i \in \mathbb{Z}}$. By construction, the two sequences consist of positive integers, and $\nu$ is the smallest common period. Also by construction,

$$
n=n_{1}+\ldots+n_{\nu}, \quad k=h+n,
$$

where

$$
h=h_{1}+\ldots+h_{\nu} .
$$

Given $\left(t_{0}, \ldots, t_{\nu-1}\right) \in \mathbb{R}^{\nu}$ and $\left(u_{1}, \ldots, u_{\nu-1}\right) \in \mathbb{R}^{\nu-1}$, put

$$
\begin{aligned}
\psi_{2 \nu-1}\left(t_{0}, u_{1}, t_{1}, u_{2}, \ldots, t_{\nu-1}\right) & \\
& =\sum_{l=0}^{\nu-1} 2^{t_{0}+u_{1}+\ldots+t_{l-1}+u_{l}}\left\{2^{t_{l}}-1\right\} 3^{u_{l+1}+\ldots+u_{\nu-1}},
\end{aligned}
$$

where the sum in the 2-exponent (3-exponent) is zero if $l=0(l=\nu-1)$. For each $j \in \mathbb{Z}$ define

$$
H_{j}=\psi_{2 \nu-1}\left(h_{j}, n_{j+1}, h_{j+1}, n_{j+2}, \ldots, h_{j+\nu-1}\right) .
$$

Thus $H_{j}$ does not depend on $n_{j}$. Note that $H_{j}>0$ for each $j \in \mathbb{Z}$. Note also that $\left\{H_{j}\right\}$ is $\nu$-periodic. We assert

$$
\widetilde{F}_{\tau(j)}=2^{n_{j+1}} H_{j+1} \quad(j \in \mathbb{Z}) .
$$

By shift, it is enough to verify (3.7) for $j=0$. Also by shift, we can assume that $\tau(0)=0$. Then $\tau(j)$ equals $n_{1}+\ldots+n_{j}(1 \leq j \leq \nu)$. In (3.1) only those terms can survive which are placed at $l+1=\tau(j)$. Hence

$$
\widetilde{F}_{\tau(0)}=2 \sum_{j=1}^{\nu} 3^{n-\tau(j)} 2^{h_{1}+\ldots+h_{j-1}+\tau(j)-1}\left\{2^{h_{j}}-1\right\} .
$$

In the last formula we take the 2-exponent $n_{1}-1$ outside the sum. After some rearrangement, the remaining sum is easily identified as $H_{1}$.

Conversely, let $\left\{h_{j}\right\}_{j \in \mathbb{Z}},\left\{n_{j}\right\}_{j \in \mathbb{Z}}$ be any periodic sequences consisting of positive integers. Let $\nu$ be the smallest common period of both sequences. Define $h, k, n$ by (3.3) and (3.4). Let $H_{j}$ be given by (3.6). Up to shift, $\left\{k_{i}\right\}$ is uniquely determined by both sequences, i.e. by $\left\{h_{0}, n_{1}, h_{1}, n_{2}, \ldots\right\}$. Also $n$ is the smallest period of $\left\{k_{i}\right\}$. Because of $(2.8),(3.2)$ and (3.7), we can reformulate Lemma 2.1. 
Lemma 3.1. Let $M=2^{k}-3^{n}$. Then either $M \nmid H_{j}(1 \leq j \leq \nu)$ or $M \mid H_{j}(1 \leq j \leq \nu)$. The latter case corresponds to a Collatz cycle with $|\Gamma|=n$ and $\delta(\Gamma)=\nu$.

4. Minimum of the $H$-sequence. In this section we prove Theorem 1.1 assuming the truth of Lemma 5.1, which will be stated and proved in

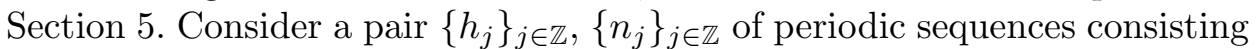
of positive integers. Define $\nu, h, k, n$ as described in the preceding section. By Lemma 3.1, since each $H_{j}>0$, the two sequences cannot generate a Collatz cycle if

$$
\min _{0 \leq j \leq \nu-1} H_{j}<|M|=\left|2^{k}-3^{n}\right| .
$$

To prove Theorem 1.1, it is enough, by Lemma 2.2 and Remark 2.3, to show (4.1) holds for all sufficiently large $n$, assuming that $\nu<2 \log n$.

First we consider the left hand side of (4.1). Let $\theta=\log _{2} 3$ and $\theta_{1}=\theta-1$. Note that $\theta_{1}>0$. In order to estimate $H_{j}$, we replace the bracket inside (3.5) by $2^{h_{j+l}}$. Because of periodicity, an easy rearrangement yields

$$
3^{-n} H_{j}<\sum_{l=0}^{\nu-1} 2^{-n_{j}+S_{j, l}} \quad(j \in \mathbb{Z})
$$

where

$$
S_{j, l}=\sum_{i=0}^{l}\left(h_{j+i}-\theta_{1} n_{j+i}\right) \quad(l \geq 0) .
$$

As a functional of both sequences define

$$
E=\min _{0 \leq j \leq \nu-1}\left\{-n_{j}+\max _{0 \leq l \leq \nu-1} S_{j, l}\right\} .
$$

By an elementary estimation,

$$
\min _{j} 3^{-n} H_{j}<\nu 2^{E}
$$

We claim that

$$
E \leq \max (0, \Delta)-A_{\nu} n,
$$

where

$$
A_{\nu}=\theta_{1} /\left(\theta^{\nu}-1\right), \quad \Delta=h-\theta_{1} n=k-\theta n .
$$

But (4.2) is a consequence of Lemma 5.1 below, applied with $x_{j}=\theta_{1} n_{j}$, $y_{j}=h_{j}, \alpha=1 / \theta_{1}$. Hence we obtain

$$
\min _{j} 3^{-n} H_{j}<\nu 2^{\max (0, \Delta)-A_{\nu} n} .
$$

Let $\gamma=1-2 \log \theta=0.0788 \ldots$ If we assume that $\nu<2 \log n$, then

$$
\left(\theta^{\nu}-1\right)^{-1} n>\theta^{-\nu} n=n^{1-\nu \log \theta / \log n}>n^{\gamma},
$$


and it follows that

$$
\min _{j} 3^{-n} H_{j}<\log n \cdot 2^{1+\max (0, \Delta)-\theta_{1} n^{\gamma}} .
$$

We now consider the right hand side of (4.1). By elementary analysis we get

$$
3^{-n}|M|=\left|2^{\Delta}-1\right| \geq \min (|\Delta|, 1) 2^{\max (0, \Delta)-1} .
$$

Note that we have $n \geq 1$ and $k=h+n \geq 2$. By definition, $|\Delta|<1$ if $k>\theta n-1$ and $k<\theta n+1$, otherwise $|\Delta| \geq 1$. A result of A. Baker and N. Feldman (see Theorem 3.1 in [1]) implies the existence of an effectively computable constant $C_{0}>0$ such that

$$
|\Delta|>|k \log 2-n \log 3|>\{\max (k, n)\}^{-C_{0}} \quad(k \geq 2, n \geq 1) .
$$

Since $\theta n+1>n$,

$$
|\Delta|>\{\theta n+1\}^{-C_{0}},
$$

and therefore

$$
3^{-n}|M|>\{\theta n+1\}^{-C_{0}} 2^{\max (0, \Delta)-1} .
$$

If $n$ is large enough, the right side of (4.3) is smaller than the right side of (4.4), which gives (4.1).

REMARK 4.1. We briefly sketch the extension of Theorem 1.1 to the $3 x+d$ mapping, where $d$ is a positive integer prime to 2 and 3 . Define $T_{1}$ according to (1.1), where the right hand side is replaced by $3 x+d$. Now $k(x)$ is the multiplicity of 2 in the number $3 x+d$. Define a Collatz cycle, descending and $\delta(\Gamma)$ as in Section 1. After an obvious modification of (2.1), in (2.2) replace $\varphi_{m-1}$ by $d \varphi_{m-1}$. Note that the definition of $\varphi_{m}$ is not affected $(m \geq 0)$. Hence multiply the right hand side of (2.5) with $d$. If (2.6) is multiplied with $d$, it follows that $F_{i}$ can be replaced by $d F_{i}$ in Lemma 2.1. Similarly to the proof of Lemma 2.2, if $\left\{k_{i}\right\}$ generates a Collatz cycle, then

$$
2^{k}=\prod_{i=1}^{n}\left(3+d / x_{i-1}\right) \leq(3+d)^{n} .
$$

Hence put $k \leq C_{1} n$ in Lemma 2.2, where $C_{1}=\log _{2}(3+d)$. In Lemma 3.1 put $d H_{j}$ instead of $H_{j}$. Finally multiply the left hand side of (4.1) with $d$. The additional factor $d$ causes no harm for the conclusion that the inequality is true if $n$ is large enough and $\nu<2 \log n$.

5. Upper value of $E$. Let there be given an integer $\nu \geq 1$ and real numbers $r>0, s>0$. Let $\left\{x_{j}\right\}_{j \in \mathbb{Z}},\left\{y_{j}\right\}_{j \in \mathbb{Z}}$ be $\nu$-periodic sequences of non-negative real numbers such that

$$
x_{1}+\ldots+x_{\nu}=r, \quad y_{1}+\ldots+y_{\nu}=s .
$$


For each $j \in \mathbb{Z}$ and $l \geq 0$ define

$$
S_{j, l}=\sum_{i=0}^{l}\left(y_{j+i}-x_{j+i}\right) .
$$

Lemma 5.1. For any real $\alpha>0$,

$$
\min _{0 \leq j \leq \nu-1}\left\{-\alpha x_{j}+\max _{0 \leq l \leq \nu-1} S_{j, l}\right\} \leq \max (0, s-r)-r /\left(\beta^{\nu}-1\right),
$$

where $\beta=1+1 / \alpha$.

Proof. (a) Reduction to the case $r=s$. If $r \neq s$, put $\widetilde{y}_{j}=r y_{j} / s$. Then $\widetilde{s}=r$ and

$$
S_{j, l}=\sum_{i=0}^{l}\left(y_{j+i}-\widetilde{y}_{j+i}\right)+\sum_{i=0}^{l}\left(\widetilde{y}_{j+i}-x_{j+i}\right)=\frac{s-r}{s} \sum_{i=0}^{l} y_{j+i}+\widetilde{S}_{j, l} .
$$

Hence

$$
\max _{0 \leq l \leq \nu-1} S_{j, l} \leq \max (0, s-r)+\max _{0 \leq l \leq \nu-1} \widetilde{S}_{j, l} .
$$

Thus we have to prove the assertion for the case $r=s$.

(b) Elimination of $\left\{y_{j}\right\}$. We suppose $r=s$. Then $\left\{S_{0, l}\right\}_{l \geq 0}$ is $\nu$-periodic. By a simple shift, we can assume

$$
S_{0, \nu-1}=\max _{l \geq 0} S_{0, l}=0 .
$$

If $1 \leq j \leq \nu-1$, then

$$
\max _{0 \leq l \leq \nu-1} S_{j, l}=\sum_{i=j}^{\nu-1}\left(y_{i}-x_{i}\right)=\sum_{i=0}^{j-1}\left(x_{i}-y_{i}\right) \leq \sum_{i=0}^{j-1} x_{i} .
$$

Note that the last inequality is sharp if $y_{j}=0(0 \leq j \leq \nu-2)$ and $y_{\nu-1}=r$. Now we have to estimate the minimum of

(c) Linear optimization. Consider $x=\left(x_{0}, \ldots, x_{\nu-1}\right)$ as a vector in $\mathbb{R}^{\nu}$. Define linear functionals $L_{0}, \ldots, L_{\nu-1}$ according to (5.1). Let $f(x)$ be the minimum of $L_{0} x, \ldots, L_{\nu-1} x$. Let $B$ be the affine subspace $x_{0}+\ldots+x_{\nu-1}=r$. We assert

$$
\sup _{x \in B} f(x)=-r /\left(\beta^{\nu}-1\right) .
$$

In order to prove (5.2), consider the vector

$$
b=r(\beta-1)\left(\beta^{\nu}-1\right)^{-1}\left(\beta^{0}, \beta^{1}, \ldots, \beta^{\nu-1}\right) .
$$

Plainly $b \in B$. An easy computation shows

$$
L_{0} b=L_{1} b=\ldots=L_{\nu-1} b .
$$


Next, $f(b)$ equals the right hand side of (5.2). Let $U$ be the linear subspace where the sum of coordinates equals zero. If $x \in B$, then $x=b+u(u \in U)$. By (5.3),

$$
f(x)=f(b)+\min _{j} L_{j} u=f(b)+f(u) \quad(x \in B) .
$$

We have to show $f(u) \leq 0$. Assume the contrary, that is, each number $L_{0} u, \ldots, L_{\nu-1} u$ is positive. Since $L_{0} u>0$, we get $u_{0}<0$. Since $u_{0}<0$ and $L_{1} u>0$, we get $u_{1}<0$. Repeating the argument, each $u_{j}$ is negative, which contradicts $u \in U$.

Acknowledgements. We thank the referee for useful hints and improvements.

\section{References}

[1] A. Baker, Transcendental Number Theory, Cambridge Univ. Press, 1975.

[2] E. G. Belaga and M. Mignotte, Embedding the $3 x+1$ conjecture in a $3 x+d$ context, Experiment. Math. 7 (1998), 145-152.

[3] L. Halbeisen and N. Hungerbühler, Optimal bounds for the length of rational Collatz cycles, Acta Arith. 78 (1997), 227-239.

[4] J. C. Lagarias, The $3 x+1$ problem and its generalizations, Amer. Math. Monthly 92 (1985), 3-23.

[5] - , The set of rational cycles for the $3 x+1$ problem, Acta Arith. 56 (1990), 33-53.

[6] R. P. Steiner, A theorem on the Syracuse Problem, in: Proc. 7th Manitoba Conf. on Numerical Mathematics, 1977, Winnipeg, 1978, 553-559.

[7] G. J. Wirsching, The Dynamical System Generated by the $3 n+1$ Function, Lecture Notes in Math. 1681, Springer, Berlin, 1998.

Helblingstr. 21

D-70565 Stuttgart, Germany

E-mail: thomas.brox@daimlerchrysler.com 